\title{
A difusão do câncer de mama em mulheres na mídia impressa
}

\author{
The diffusion of breast cancer in women in print media
}

\author{
Shirley de Souza Silva Simeão ${ }^{1}$ \\ Maria da Penha de Lima Coutinho² \\ Lidiane Silva de Araújo 3 \\ Rosane de Sousa Miranda ${ }^{4}$
}

\begin{abstract}
RESUMO: Objetivou-se apreender as representações sociais acerca do câncer de mama em mulheres difundidas pelas mídias impressas, nacional (Folha de São Paulo) e local (Correio da Paraíba). Realizou-se um estudo documental com base em 58 matérias jornalísticas veiculadas em 2012 e 2013. Os dados foram processados por meio da análise padrão no Alceste, a partir da qual identificaram-se quatro classes temáticas. Em geral, os resultados evidenciaram a representação da doença como prevenível, ao mesmo tempo em que focalizaram o caratér fatal da doença. Embora a comunicação midiática seja um importante fio condutor de informações sobre a neoplasia mamária, os resultados indicaram a necessidade de questionar sobre a natureza do conteúdo que é enfaticamente veiculado pelos jornais. Por exemplo, a mídia não apontou a adoção de um estilo de vida saudável como conduta preventiva igualmente pertinente, valorizando hegemonicamente o enfoque médico (realização de exames periódicos). Além disso, ao contrário do que se esperava, a ênfase em notícias com cifras sobre mortalidade e outras considerações científicas pode ter um efeito repulsivo para práticas de autocuidado e detecção precoce da doença, uma vez que a doença fica associada à morte como realidade próxima, contribuindo para cristalizar o estigma associado à representação do câncer de mama. Os achados serviram para explicar a difusão do câncer de mama pela mídia impressa, tornando notória a necessidade de inclusão de pautas de comunicação positivas sobre o tema e, suas possíveis implicações nas práticas de prevenção e cuidado no contexto oncológico.
\end{abstract}

Palavras-chave: câncer de mama; mulheres; mídia impressa; difusão; representações sociais.

ABSTRACT: This study aimed to identify the social representations of breast cancer in women broadcast by national print media (Folha de São Paulo) and local (Correio da Paraíba). We conducted a documental study based on 58 journalistic articles published in 2012 and 2013. Data was analysed with the software Alceste, using the default procedure, which allowed to identify a corpus organized in four thematic classes. The results showed the representation of the disease as preventable, while focused on the fatal nature of the disease. Although media communication is an important thread of information about breast cancer, the results indicate the need to question about the nature of the content that is emphatically conveyed by newspapers. For example, the media did not point the adoption of a healthy lifestyle as equally relevant preventive conduct, valuing hegemonically medical approach (periodic examinations). Moreover, contrary to what was expected, the emphasis on news to figures on mortality and other scientific considerations may have a repulsive effect on self-care practices and early detection of disease, since the disease is associated with death as close reality, contributing to crystallize the stigma associated with the representation of breast cancer. The findings served to explain the diffusion of the breast cancer by the print media, making evident the need for inclusion of positive communication guidelines on the issue and its possible implications for prevention and care practices in the oncological context.

\footnotetext{
${ }^{1}$ Doutora em Psicologia Social. Professora adjunta da Faculdade Maurício de Nassau - São Luís, MA, Brasil. E-mail: shirley.psic@yahoo.com.br.

2 Doutora em Psicologia Clínica. Professora emérita da Universidade Federal da Paraíba - João Pessoa, PB, Brasil.

${ }^{3}$ Mestre em Psicologia Social. Doutoranda em Psicologia Social pela Universidade Federal da Paraíba - João Pessoa, PB, Brasil.

${ }^{4}$ Doutora em Psicologia Social. Professora adjunta da Universidade Federal do Maranhão - São Luís, MA, Brasil.
} 
Keywords: breast cancer; women; print media; diffusion; social representations.

\section{Introdução}

Por ser considerado uma das primeiras causas de mortalidade no mundo (RodríguezSales, Ortiz-Barreda \& Sanjosé, 2014), o câncer de mama tem sido um assunto presente no cotidiano social, recebendo lugar de destaque na comunicação que é veiculada pelas mídias.

Nos últimos anos, segundo o Instituto Nacional do Câncer (INCA, 2014), a população mundial se deparou com o aumento de $22 \%$ no número de casos de câncer de mama. De acordo com os dados publicados pelo instituto, a cada ano aumenta a incidência de casos e de mortalidade pela doença, chegando a 57 mil o número de novos casos de câncer de mama entre as mulheres. Apesar de ser considerado prevenível e dos avanços científicos empreendidos para a sua detecção e tratamento, este problema de saúde pública é o segundo tipo de neoplasia mais incidente e o mais prevalente entre as mulheres (Inumaru, Silveira \& Naves, 2011; Kluthcovsky, Faria, Carneiro \& Strona, 2014; Soares et al, 2013), pois ainda é concebido como uma sentença de morte, realidade que demanda atenção prioritária das políticas públicas de saúde nesta esfera.

De acordo com Giraldo-Mora (2009), a expressão câncer de mama traz uma forte associação ao sofrimento, à impotência, à dor e à morte inevitável. Tal representação se deve, especialmente, às implicações e repercussões que a patologia representa para a figura feminina e sua autoimagem. Ao incidir sob a mama, símbolo da feminilidade e sensualidade, o câncer afeta o psiquismo e a relação da mulher com seu corpo, alterando sua qualidade de vida em múltiplas dimensões.

Com efeito, a representação social (RS) que a doença assume socialmente tem implicações práticas nos comportamentos adotados pelo indivíduo na situação de adoecimento e, por conseguinte, no seu modo de tratar a doença (Oliveira, 2011; Silva, Bousfield \& Cardoso, 2014). Assim, acessar o saber que a população possui sobre determinada doença e suas formas de prevenção, pode constituir um caminho possível para pensar e fundamentar a implementação de campanhas preventivas eficazes (Quintana et al., 2004).

Nesse direcionamento, a mídia funciona como um vetor importante para o saber social que é produzido acerca de uma doença específica ou um dado objeto representacional (Silva et al., 2014). De acordo com Moscovici (2011), são estas influências dos processos de comunicação que dão sustentáculo às RS, constituindo as realidades típicas da vida social. Portanto, torna-se mister apreender o tipo de RS do câncer de mama que é comumente veiculado pela mídia; afinal, conhecer os conteúdos que circulam no tecido social por meio de notícias jornalísticas pode dar uma dimensão dos esquemas de pensamento e de comportamento que são associados ao tema e possivelmente perpassam o decurso do cotidiano dos atores sociais.

Considerando que a informação pode viabilizar a promoção da educação e a garantia do acesso aos direitos da saúde feminina, a mídia, em seu papel de difusão de informações do saber científico para o senso comum (Saraiva \& Coutinho, 2012a), pode contribuir para a promoção de saúde, auxiliando na prevenção e diminuição da mortalidade por câncer de mama (Feldman, 2008).

De acordo com Coutinho e Saraiva (2013), o processo de construção das RS está vinculado diretamente às ações de comunicação, o que confere a esta o papel de pedra 
angular das RS, tendo em vista que ela (a comunicação) não se refere à mera transmissão de informação, mas corresponde a uma via que pode favorecer a ressignificação da mensagem que vai sendo alterada a partir de normas e valores coletivos (Moscovici, 2012; Vala, 2013).

$\mathrm{Na}$ condição de teoria do conhecimento social, a teoria das RS propõe-se a apresentar e discutir o processo pelo qual o conteúdo do conhecimento científico se transforma, dando lugar a um novo tipo de conhecimento: o conhecimento do senso comum (Moscovici, 2011; Oliveira, 2011). Para tanto, esse processo de popularização ou apropriação de um conhecimento se desenvolve a partir de três sistemas de comunicação (difusão, propagação e propaganda) que vão determinar o conteúdo e a forma das mensagens emitidas e recebidas. Tais sistemas estão relacionados a dimensões peculiares das RS, ligados a áreas específicas que vão desde a formação das opiniões (difusão), das atitudes (propagação) aos estereótipos (propaganda) (Coutinho \& Saraiva, 2013; Jodelet, 2001; Moscovici, 2012).

Os meios de comunicação difundem e expressam elementos representacionais dominantes do contexto sociocultural, contribuindo com a transmissão de conhecimentos científicos e funcionando como instrumento de convencimento e disseminação de RS. Cada vez mais evidencia-se que a mídia assume um papel central na formação da imagem de uma determinada realidade/objeto, a partir do conteúdo transmitido/veiculado (Allain \& Camargo, 2007; Saraiva, 2010).

Desta feita, a informação passa a ser considerada uma importante aliada no combate à doença (Jurberg, Gouveia \& Belisário, 2006). Entretanto, neste cenário, o câncer tem sido frequentemente representado como algo negativo, invasivo, que remete ao medo, à dor e à possibilidade de morte iminente, sendo considerada uma doença perigosa, sinônimo de sofrimento e fatalidade. Acessar as RS que circulam acerca da doença colabora para objetivação do contexto social da saúde e a reconfiguração de objetos e intervenções, favorecendo e direcionando comportamentos e ações de autocuidado (Giraldo-Mora, 2009; Tavares \& Trad, 2005).

Segundo Saraiva e Coutinho (2012b) a mídia, principalmente os jornais, tem sido um veículo das RS, à medida que, por meio de uma linguagem habitual, tem tornado acessível fatos da realidade diária, contribuindo para a formação e difusão das RS nas diversas esferas da sociedade. Com base nestas considerações, esta pesquisa apresenta-se com o desígnio de desvelar a imagem midiática associada à referida patologia, objetivando, especificamente, apreender as RS acerca do câncer de mama que são disseminadas pela mídia impressa. Conforme Moscovici (2012), a qualidade e o tipo de informações acerca do objeto social influenciam a formação das RS. Dessa forma, como difusora de RS, a mídia assume a função de dirigir-se a um grupo difuso com o objetivo de informar, criando nesse grupo de pertença um interesse comum sobre determinado assunto (Silva et al., 2014).

Quanto à neoplasia mamária - construto dotado de espessura social - Castro (2009) afirma que a mídia tem um posto fundamental na disseminação de informações sobre o câncer, pois informações de qualidade contribuem para conscientizar a população sobre a importância do diagnóstico precoce e da adoção de hábitos saudáveis para prevenção da doença. No Brasil, ao alertar a população com informações relevantes, sobre a importância da prevenção e do diagnóstico precoce do câncer de mama entre as mulheres, a publicação sobre o assunto nos meios de comunicação pode constituir uma forma de colaborar embora não necessariamente de maneira determinante - para a prevenção e diminuição do número de mortes em função da doença. 
Face ao exposto, torna-se perceptível a relevância social e acadêmica da presente proposta de pesquisa, que objetivou conhecer as representações sociais da mídia impressa sobre o câncer de mama em mulheres. Indubitavelmente, o acesso a esse tipo de conhecimento pode servir para situar a importância da mídia impressa na difusão de informações sobre o tema, identificando os possíveis eixos de discussão sobre o assunto e, de algum modo, evidenciar as lacunas temáticas, orientando novos avanços na comunicação. Em outras palavras, espera-se que esta investigação oportunize o conhecimento sobre os elementos de prevenção do câncer de mama e de subsídios para a construção de práticas preventivas no contexto da comunicação e sua interface com a oncologia.

\section{Método}

\section{Tipo de estudo e caracterização dos documentos}

Trata-se de uma pesquisa documental, de natureza descritiva e exploratória, realizada a partir da coleta de notícias veiculadas na mídia impressa sobre o câncer de mama entre mulheres, disponíveis em portais eletrônicos dos jornais Folha de São Paulo e Correio da Paraíba. Quanto à escolha dos jornais, seguiram-se os critérios propostos por Saraiva, Coutinho e Miranda (2011), isto é, incluir jornais de grande circulação, com representativa tiragem, de alcance nacional e estadual, e disponíveis em formato eletrônico. Para a varredura, foram consideradas as matérias jornalísticas veiculadas no período de janeiro de 2012 a junho de 2013, período determinado pelo grande volume de publicações envolvendo a temática. Vale salientar que a faixa temporal referida foi intencionalmente determinada em função da constatação de um grande volume de publicações sobre o tema envolvendo um caso de câncer de mama de uma celebridade, realidade que conferiu, no período aludido, um robusto destaque ao assunto da neoplasia mamária pela mídia.

\section{Procedimento}

A leitura e seleção dos artigos foram realizadas nos sites dos jornais, utilizando-se o descritor "câncer de mama". Dos 170 artigos encontrados a partir da busca inicial, foram mantidos 58, cujo foco estava diretamente relacionado ao câncer de mama em mulheres, excluindo-se, portanto, as matérias que apenas citavam o termo e que não estavam relacionadas ao contexto feminino propriamente dito. Em seguida, cada matéria selecionada foi submetida à análise de juízes a fim de avaliar a sua adequação aos critérios supramencionados; ao final, as 58 matérias selecionadas foram transferidas para um arquivo de texto.

\section{Análise dos dados}

O conjunto das notícias constituiu um corpus que foi processado no software ALCESTE (Analyse Lexicale par Contexte d'un Ensemble de Segments de Texte), por meio da análise lexical padrão. Desenvolvido por Reinert, o programa informático apoia-se em cálculos efetuados sobre a frequência e qui-quadrado de palavras nos segmentos de texto, buscando distinguir classes de palavras que representem formas distintas de discurso sobre os tópicos 
de interesse da investigação, facilitando a análise psicossocial (Saraiva \& Coutinho, 2012b; Saraiva, Coutinho \& Miranda, 2011) de um tema, neste estudo, do câncer de mama.

As notícias selecionadas foram armazenadas em um único banco de dados (corpus textual monotemático), a fim de verificar os conteúdos veiculados sobre o câncer de mama pelos distintos jornais. Portanto, a linha de comando considerou os tipos de jornais, anos de publicação e focos das matérias, que determinaram a constituição das linhas de comando. Em seguida, os dados foram submetidos à análise padrão desempenhada pelo software (classificação hierárquica descendente).

\section{Resultados}

A partir dos documentos estudados, após a seleção das matérias publicadas no período de busca anteriormente descrito, foi possível observar que o jornal Folha de São Paulo apresentou 44 notícias relacionadas diretamente ao câncer de mama em mulheres, enquanto o jornal Correio da Paraíba apresentou apenas 14 notícias; possivelmente, este número reduzido de publicações indica que o tema de interesse pode não estar recebendo a devida atenção na mídia local.

Além da notória diferença entre o volume de publicações, foi evidenciado também que no jornal local (Correio da Paraíba), das 14 notícias selecionadas, sete tiveram como foco o diagnóstico e medidas de prevenção, duas abordaram dados epidemiológicos, trazendo informações sobre incidência da doença, duas evidenciaram aspectos relacionados ao tratamento e três abordaram o câncer de mama como assunto secundário ao conteúdo principal da notícia. Neste último caso, a notícia secundária se deu na forma de nota de falecimento de uma figura representativa na sociedade, bem como pela divulgação de algum evento, peça teatral ou livro, sobre o tema.

Do conjunto de 44 notícias veiculadas pelo Jornal Folha de São Paulo, 16 abordaram ações de diagnóstico e prevenção, 17 trouxeram o tema câncer de mama como secundário à informação transmitida, 10 agruparam informações relacionadas à terapêutica da doença, como descobertas e ações de novos medicamentos, e apenas uma abordou dados epidemiológicos, incidência de novos casos e registro de mortalidade pela doença.

O material textual decorrente da pesquisa documental realizada nos jornais permitiu a formação de um corpus que foi submetido ao tratamento padrão desenvolvido pelo Alceste, processo que tomou por base um corpus composto por 58 unidades de contexto iniciais (UCls) que, por sua vez, repreentavam as 58 notícias selecionadas para análise.

O corpus foi dividido em 393 unidades de contextos elementares (UCEs), inteirando 14.654 ocorrências de palavras, que, após a realização da redução dos vocábulos às suas raízes, resultou em 3.548 formas distintas, com uma média de 4 ocorrências por palavra. Neste processo, $89 \%$ das UCEs foram retidas no corpus (437), as quais foram distribuídas em quatro classes. Estas, por seu tempo, foram formadas com, no mínimo, 20 UCEs, conforme pode ser visualizado no dendrograma representado na Figura 1. 


\begin{tabular}{|c|c|c|c|c|c|c|c|c|c|c|c|}
\hline \multicolumn{12}{|c|}{ ASPECTOS RELACIONADOS À PREVENCÃO E TRATAMENTO DO CÂNCER DE MAMA } \\
\hline & \multicolumn{6}{|c|}{ Discutindo as possibilidades terapêuticas } & \multicolumn{5}{|c|}{$\begin{array}{l}\text { Principais ações relacionadas à } \\
\text { prevenção do câncer de mama }\end{array}$} \\
\hline & & & & & & & & & & & \\
\hline \multicolumn{2}{|l|}{58 UCE } & & $\begin{array}{r}\text { CLASS } \\
108 \mathrm{U} \\
31 \%\end{array}$ & & & $\begin{array}{r}\text { CLASSE } \\
162 \text { UC } \\
46 \%\end{array}$ & & & $\begin{array}{c}\text { CLASSE } \\
20 \text { UCE } \\
6 \%\end{array}$ & & \\
\hline \multicolumn{3}{|c|}{$\begin{array}{l}\text { Diretrizes governamentais } \\
\text { e científicas para o } \\
\text { tratamento } \\
\text { medicamentoso do câncer } \\
\text { de mama }\end{array}$} & \multicolumn{3}{|c|}{$\begin{array}{l}\text { Predisposição genética ao } \\
\text { câncer de mama: } \\
\text { consequências e } \\
\text { possibilidades terapêuticas }\end{array}$} & \multicolumn{3}{|c|}{$\begin{array}{l}\text { Estudos e ações voltados } \\
\text { para a prevenção e } \\
\text { promoção da saúde da } \\
\text { mama da mulher }\end{array}$} & \multicolumn{3}{|c|}{$\begin{array}{l}\text { Principais ações envolvidas } \\
\text { nas campanhas de } \\
\text { prevenção }\end{array}$} \\
\hline Palavras & $\chi^{2}$ & $f$ & Palavras & $\chi^{2}$ & $f$ & Palavras & $\chi^{2}$ & $f$ & Palavras & $\chi^{2}$ & $F$ \\
\hline Remédio & 78 & 22 & Atriz & 51 & 23 & Mulher & 26 & 87 & Outubro rosa & 186 & 11 \\
\hline Trastuzumabe & 62 & 13 & Cirurgia & 44 & 31 & Pode & 23 & 41 & Palestra & 100 & 6 \\
\hline $\begin{array}{l}\text { Ministério da } \\
\text { saúde }\end{array}$ & 51 & 10 & Mastectomia & 38 & 23 & Exame & 16 & 25 & Sistema Correio & 100 & 6 \\
\hline Governo & 50 & 13 & Angelina Jolie & 37 & 25 & Precoce & 14 & 13 & Importância & 88 & 7 \\
\hline Médica & 45 & 12 & Descobrir & 31 & 15 & Tumores & 13 & 25 & Iluminação & 83 & 5 \\
\hline Ministério & 41 & 8 & Mãe & 30 & 16 & Diagnóstico & 13 & 24 & Prédio & 83 & 5 \\
\hline Incorporação & 31 & 6 & Dupla & 21 & 9 & Estados Unidos & 12 & 19 & Atividades & 83 & 6 \\
\hline Rompida & 25 & 6 & Disse & 19 & 10 & Ajudar & 12 & 10 & Associação & 66 & 5 \\
\hline Justiça & 25 & 5 & Filha & 19 & 11 & Tipo & 12 & 10 & Prevenção & 58 & 8 \\
\hline Rede pública & 25 & 7 & Procedimento & 18 & 12 & Dos & 11 & 38 & Comunicação & 52 & 4 \\
\hline Droga & 21 & 10 & Aos & 15 & 13 & Estudo & 11 & 17 & \begin{tabular}{|l}
$\begin{array}{l}\text { Saúde } \\
\text { mulher }\end{array}$ \\
\end{tabular} & 52 & 4 \\
\hline Marcas & 20 & 5 & Fez & 15 & 11 & Importante & 11 & 10 & Funcionários & 50 & 4 \\
\hline Troca & 20 & 6 & Seio & 15 & 14 & Estudos & 10 & 12 & Campanha & 42 & 6 \\
\hline Usado & 20 & 8 & Uma & 14 & 58 & País & 10 & 17 & Sede & 36 & 3 \\
\hline Oferta & 20 & 4 & Vez & 12 & 9 & Parte & 10 & 18 & Aderiu & 36 & 3 \\
\hline ANVISA & 20 & 4 & Deixou & 12 & 7 & Teste & 10 & 24 & Estimular & 36 & 3 \\
\hline Efeito & 20 & 5 & Deus & 11 & 5 & Risco & 10 & 38 & Objetivo & 36 & 3 \\
\hline Distribuição & 20 & 4 & Dias & 11 & 12 & Maior & 9 & 18 & Realização & 23 & 6 \\
\hline Rede privada & 20 & 4 & Morreu & 11 & 9 & Mostra & 9 & 12 & Rosa & 22 & 4 \\
\hline Uso & 18 & 7 & Remover & 11 & 5 & Brasil & 9 & 28 & Mês & 17 & 4 \\
\hline Avançado & 18 & 7 & Retirada & 11 & 12 & Brca 2 & 9 & 8 & Acontecerá & 15 & 3 \\
\hline Compra & 15 & 3 & Filhos & 11 & 5 & Identificar & 9 & 9 & Atenção & 15 & 2 \\
\hline Científico & 15 & 4 & Teve & 9 & 8 & Mama & 8 & 23 & Auto-exame & 15 & 3 \\
\hline Oncoguia & 15 & 4 & Devido & 9 & 4 & Idade & 8 & 7 & População & 15 & 4 \\
\hline Everolimo & 15 & 4 & Mamilo & 9 & 11 & Mamografia & 8 & 18 & Ganha & 13 & 3 \\
\hline Substituição & 15 & 4 & Aréola & 8 & 6 & Lesão & 7 & 6 & Nesta & 13 & 3 \\
\hline Grupo & 14 & 7 & Ficava & 8 & 10 & Mutações & 7 & 17 & $\mathrm{Da}$ & 12 & 27 \\
\hline \multicolumn{3}{|c|}{ Jornal: Folha de São Paulo } & \multicolumn{3}{|c|}{$\begin{array}{l}\text { Jornais: Folha de São Paulo e } \\
\text { Correio da Paraíba }\end{array}$} & \multicolumn{3}{|c|}{ Jornal: Folha de São Paulo } & \multicolumn{3}{|c|}{ Jornal: Folha de São Paulo } \\
\hline \multirow{2}{*}{\multicolumn{3}{|c|}{ Foco da notícia: tratamento }} & \multicolumn{3}{|c|}{ Ano: 2013} & \multicolumn{3}{|c|}{ Ano: 2012} & \multicolumn{3}{|c|}{ Ano: 2012} \\
\hline & & & \multicolumn{3}{|c|}{$\begin{array}{l}\text { Foco da notícia: câncer de } \\
\text { mama como tema secundário }\end{array}$} & \multicolumn{3}{|c|}{$\begin{array}{l}\text { Foco da notícia: dados } \\
\text { epidemiológicos }\end{array}$} & $\begin{array}{r}\text { Foco da notícia: } \mathrm{p} \\
\text { diagnósti }\end{array}$ & evençã & \\
\hline
\end{tabular}

Figura 1 - Dendrograma da análise hierárquica descendente das notícias sobre câncer de mama. 
O corpus denominado "Aspectos relacionados à prevenção e tratamento do câncer de mama de mulheres" apresentou, a partir da análise da Classificação Hierárquica Descendente (CHD), em sua primeira partição, uma divisão composta por quatro classes distintas, as quais foram agrupadas em dois subcorpora. Localizado à esquerda, o subcorpus que determinou as classes 1, 2 e 4 foi denominado de "Discutindo as possibilidades terapêuticas"; o subcorpus localizado à direita do dendrograma, que determinou a classe 3 , foi denominado de "Principais ações relacionadas à prevenção do câncer de mama".

\title{
Classe 1: Diretrizes governamentais e científicas para o tratamento medicamentoso do câncer de mama
}

Esta classe, composta por 58 UCEs (17\%), reuniu conteúdos relacionados a estudos e evidências científicas dos principais medicamentos e tratamentos efetivos nos casos de câncer de mama, os quais são disponibilizados na rede pública e privada. Além disso, as palavras associadas a esta classe também abordam ações propostas pelo governo e seus ministérios, as quais norteiam os tratamentos possíveis na rede pública.

Representada em sua maioria por notícias que têm como foco o tratamento e que foram veiculadas pelo jornal Folha de São Paulo, a classe em questão teve em sua composição palavras e radicais variando de $\chi^{2}=78$ (remédio) a 14 (grupo). Com relação ao conteúdo representativo desta classe, destacam-se os seguintes trechos de notícias:

\begin{abstract}
(...) Com a incorporação do remédio na rede pública, o governo prevê um gasto anual de 130 milhões de reais. (...) Segundo o diretor científico do Instituto Oncoguia, o novo medicamento testado ataca um alvo presente em até $25 \%$ das pacientes com câncer de mama, agindo tanto no pós operatório como nos casos em que a doença está disseminada.
\end{abstract}

\section{Classe 2: Predisposição genética ao câncer de mama: consequências e possibilidades terapêuticas}

A classe 2, composta por 108 UCEs (31\%), foi intitulada "predisposição genética ao câncer de mama: conseqüências e as possibilidades terapêuticas" por agrupar palavras que se associaram à identificação genética de predisposição ao câncer de mama, evidenciando o procedimento de mastectomia profilática como alternativa terapêutica para os casos em que há a confirmação de alterações genéticas envolvidas na doença.

Esse grupo traz, com grande representatividade, notícias relacionadas ao caso da atriz Angelina Jolie, que ganhou repercussão mundial ao declarar ter sido submetida à mastectomia dupla como estratégia para evitar o desenvolvimento futuro da doença, tendo em vista sua elevada predisposição genética à doença evidenciada por meio de exames.

Nesta classe, destacaram-se as palavras com radicais variando de $\chi^{2}=51$ (atriz) a 8 (auréola, ficava), representadas por notícias dos dois jornais considerados neste estudo, veiculadas no ano de 2013. Como dito, nesta classe tiveram destaque as matérias cujo foco principal foi a experiência vivida por uma celebridade, como mostram os recortes a seguir:

(...) Angelina Jolie afirmou que o processo de retirada dos seios começou em fevereiro. (...) A atriz explicou que sua mãe lutou contra o câncer por quase uma década e morreu aos 56 anos. (...) De acordo com ela, com o procedimento suas chances de desenvolver câncer de mama caíram de $87 \%$ para menos de 5\%. (...) Angelina Jolie anuncia ter retirado seios para evitar câncer e ter tomado essa decisão de passar por cirurgia após descobrir falha genética e surpreendeu o mundo ao anunciar que se submeteu a uma mastectomia profilática dupla. 


\section{Classe 3: Principais ações envolvidas nas campanhas de prevenção}

A presente classe contabilizou 20 UCEs (6\%), constituindo a classe que menos contribuiu com o Corpus. Sua nomeação deu-se a partir da análise das principais palavras agrupadas que remetiam às atividades desenvolvidas no mês da campanha "Outubro rosa", cujo objetivo é o incentivo à adoção de práticas preventivas e a realização de exames voltados à saúde da mama da mulher.

Neste contexto, consideraram-se as palavras com valores de $\chi^{2}$ a 186 (outubro rosa) e 12 (da), as quais foram representadas, principalmente, por reportagens veiculadas no jornal Folha de São Paulo, no ano de 2012, cujo foco foram a prevenção e o diagnóstico, conforme destacados nos excertos a seguir:

(...) Outubro rosa estimula população à prevenção do câncer de mama e iluminação especial e laço cor de rosa chamam atenção da população sobre a importância do auto-exame e da prevenção da saúde da mulher, durante todo o mês.

\section{Classe 4: Estudos e ações voltados para a prevenção e promoção da saúde da mama da mulher}

Denominada "Estudos e ações voltadas para a prevenção e promoção da saúde da mama da mulher", a classe 4 foi composta por 162 UCEs (46\%) e foi a classe que mais contribuiu com a formação do corpus. Por reunir vocábulos com valores de $\chi^{2}$ no intervalo de 26 (mulher) a 7 (lesão, mutações), estas palavras justificaram a nomeação da classe da forma como foi apresentada, contemplando ações que podem ser desenvolvidas pela mulher, como forma de proporcionar a identificação do câncer de mama em estágio inicial, favorecendo a redução da mortalidade pela patologia.

As notícias representativas desta classe foram veiculadas no ano de 2012 pelo jornal Folha de São Paulo em matérias cujo foco trazia, de forma marcante, resultados de pesquisas que apresentaram dados epidemiológicos, tais como índices de mortalidade, incidência do câncer de mama no Brasil e no mundo, além de novas pesquisas de avaliação do risco de desenvolver a doença, conforme ilustrado no trecho seguinte:

(...) Os cientistas analisaram estudos comparando os diagnósticos de câncer de mama em mulheres com e sem próteses e o trabalho indicou mulheres que podem ter maior risco de morrer por câncer de mama (...) Cientistas afirmam que só uma minoria dos tumores de mama tem origem genética, uma parcela ínfima da população carrega as mutações perigosas associadas a doença.

Conforme pode-se observar nos conteúdos representativos das classes supramencionadas, o foco das matérias aponta o câncer de mama como uma doença de alto custo relacionada majoritariamente à predisposição genética. Além disso, o tratamento foi destacado com base em ações do modelo biomédico e por meio da adoção de práticas de controle e prevenção da doença. Evidenciou-se, ainda, que a realização de campanhas preventivas importantes - como, por exemplo, o Outubro Rosa - centraliza a difusão de informações sobre a prevenção da neoplasia mamária com base na realização de exames, negligenciando outras ações preventivas igualmente relevantes.

\section{Discussão}

Conforme Castro (2009), a qualidade de informações veiculadas pela mídia pode contribuir para a conscientização das estratégias de prevenção de doenças. Considerando o 
objetivo de conhecer as representações sociais sobre o câncer de mama da mulher, veiculadas pelas mídias impressas nacional (Folha de São Paulo) e local (Correio da Paraíba), os resultados desta pesquisa evidenciaram a importância da informação midiática enquanto estratégia de mobilização para a educação em saúde, na medida que a imprensa assume um papel de destaque na disseminação de informações sobre o processo saúde/doença.

Nesse contexto, a informação é uma grande aliada na vida da mulher com câncer de mama, não só por permitir a discussão sobre aspectos relacionados à feminilidade e questões de ordem social e psicológica inerentes à vivência da doença, mas também por promover educação em saúde e alertar sobre os direitos relativos à saúde pública feminina. Com isso, oferece condições para conscientização sobre riscos e necessidades de prevenção, além de informar novas formas de abordagens terapêuticas para o câncer (Feldman, 2008; Lara, 2008).

Ao analisar as dimensões representacionais do câncer veiculadas pela imprensa, foi possível verificar que as abordagens das matérias sobre o assunto apontam para dois focos principais, os quais contemplam ações relacionadas à prevenção e ao tratamento da doença. Neste contexto, destacou-se a existência de uma representação sobre a doença em consonância com a ideia de que se trata de uma patologia prevenível a partir de ações voltadas a uma esfera biomédica e consideram, portanto, a realização de exames e o diagnóstico precoce como principais procedimentos de prevenção, em detrimento de outros fatores igualmente relevantes neste cenário.

Os resultados do presente estudo corroboram os achados de Castro (2009) acerca de matérias publicadas na mídia impressa em relação ao câncer de mama, que identificou que a maioria das reportagens publicadas detêm o foco na prevenção da doença, destacando o surgimento de novos procedimentos terapêuticos.

Assim, ao considerar as formas de comunicação propostas por Moscovici (2012), percebe-se a difusão como sistema de comunicação predominante ao se confirmar a transmissão de um conhecimento que é preciso compartilhar e que recorre à opinião de especialistas para atingir um grande público, de forma a influenciar condutas e opiniões.

Além disso, a propaganda se caracteriza como sistema de comunicação, também presente na comunicação midiática ao ter como foco o atendimento às necessidades de um grupo, em especial as mulheres, objetivando conduzi-las a uma ação, o que pode ser considerado uma função reguladora da comunicação.

Conforme Castro (2009), a mensagem evidenciada nos instrumentos de comunicação aborda o câncer como uma doença prevenível e, de maneira marcante, corrobora a ideia de que a mídia procura trabalhar o estigma de que o câncer está associado à morte, e trata-se de uma doença passível de tratamento.

Por outro lado, ao mesmo tempo em que perpassa a ideia de prevenção, os resultados convergem para a noção de que o câncer é uma patologia para a qual se tem direcionado esforços no sentido de desenvolver, de maneira urgente, a diminuição do índice de mortalidade. Tal difusão, por seu tempo, traz uma ambiguidade a ser considerada: embora seja enfatizada a busca desenfreada de meios e métodos de rastreamento da doença, o enfoque utilizado pela mídia sustenta-se majoritariamente em números, dados e argumentos que dão visibilidade ao elevado índice de mortalidade em função do câncer de mama. 
Assim, a informação veiculada acaba tendo um sentido implícito e por mais que tenha uma intenção positiva, a de informar, a estratégia voltada para a apresentação do desenvolvimento de ações e medidas preventivas acaba por reafirmar a condição do câncer como doença fatal. Logo, esta configuração no cenário midiático tende a cristalizar a representação do câncer de mama como doença perigosa, comumente associada ao sofrimento e a um desfecho fatal (Giraldo-Mora, 2009; Tavares \& Trad, 2005).

Face ao exposto, considerando que a construção e transformação das representações sociais - sustentadas pelo pilar da comunicação - devem ocorrer de forma bidirecional, vale questionar os sentidos da comunicação que perpassam a relação mídia X sociedade. Isto é, como a direção dos processos de comunicação não se dá apenas em fluxo descendente, transformando o conhecimento produzido pela ciência em senso comum, é preciso repensar a importância que a mídia dá ao saber popular. Neste aspecto, a comunicação também pode ocorrer em fluxo ascendente, transformando assuntos do senso comum em tópicos científicos. Com efeito, além de se apropriarem do conteúdo veiculado pela mídia e de adaptá-los às suas práticas, os atores sociais têm a função ativa de transformar o seu contexto social, produzindo também pautas de interesse para a mídia (Bauer, 1994; Silva et al., 2014).

Desta feita, além de dar visibilidade aos ditames científicos (dados sobre índice de mortalidade por câncer de mama e outras informações com enfoque biomédico), a mídia pode dar espaço e voz aos atores sociais, sobretudo às mulheres, grupo de pertença mais vulnerável à doença, para que falem sobre o caráter prático que a patologia assume em suas experiências diárias e como conseguem superá-la, por exemplo.

Segundo Garcia, Pereira e Marinho (2010), os mitos e medos que cercam a doença, apesar da evolução do conhecimento científico acerca do câncer, fazem com que a mídia passe a ter um papel fundamental ao divulgar avanços científicos, informar a população e derrubar os mitos de morte, desespero e solidão que cercam a enfermidade. Ao analisarem a representação construída sobre câncer veiculada pela mídia, os autores identificaram que, em algumas seções, o jornal apenas apresenta citações sobre o câncer devido a mortes, fato que acaba por reafirmar o caráter trágico da doença, contribuindo para confirmar os mitos presentes no imaginário social sobre a patologia.

Ademais, o assunto muitas vezes foi abordado por meio de pequenas notas sem fonte, que não esclarecem o real significado do achado ou informação científica, as quais visam vender uma notícia, a partir do anúncio de que determinado tipo de pessoa tem mais ou menos possibilidade de desenvolver o câncer, não conseguindo afastar-se muito da conotação negativa ou do medo da enfermidade. Deste modo, fica evidente que, embora a mídia possa colaborar para desconstruir os mitos negativos associados à doença, ela também pode corroborá-los, reforçando-os no imaginário do leitor (Garcia, Pereira \& Marinho, 2010).

Conforme Moscovici (2011), a formação das representações sociais depende da qualidade e do tipo de informações sobre o objeto social, do seu interesse pessoal sobre aspectos específicos do objeto e da influência social direcionando o indivíduo a utilizar informações predominantes no grupo. Assim, a mídia - composta por um grupo de formadores e difusores de representações sociais - é responsável pela estruturação de sistemas de comunicação que visam comunicar, difundir ou propagar determinadas representações acerca de um objeto social. 
De modo análogo, Castro (2009) preconiza que a imprensa tem um papel fundamental na disseminação de informações sobre o câncer, de modo tal que a qualidade das informações deve contribuir para conscientização da população sobre a importância do diagnóstico precoce e da adoção de hábitos saudáveis para prevenção da doença.

Segundo Lara (2008), a mídia se ocupa do assunto com regularidade, oferecendo conscientização sobre riscos e necessidade de prevenção e informando sobre novas tecnologias, novas drogas e novas abordagens no tratamento dos tumores. Cada reportagem sobre o tema suscita um grande interesse por parte da mídia, tendo em vista que os resultados e os avanços nas pesquisas em câncer são objetivos principais da área biomédica.

O tratamento que o formato eletrônico da mídia impressa brasileira disponibiliza para o tema, se bem aproveitado pela comunidade científica que investiga o câncer, pode contribuir para esclarecer sobre a patologia e se transformar em um espaço onde é possível tornar a sociedade uma aliada na prevenção dessa doença (Jurberg et al., 2006).

Outro aspecto relevante identificado no presente estudo foi que o conteúdo midiático não apontou a adoção de um estilo de vida saudável como elemento de prevenção para a doença, reconhecendo apenas as estratégias de diagnóstico precoce como possibilidades preventivas. Desta forma, considerou-se apenas o enfoque médico associado ao câncer, desprezando assim questões psicossociais envolvidas na gênese da patologia.

Tal direcionamento diverge do que é preconizado na literatura, que aponta a relação entre variáveis orgânicas e psicossociais, como fatores genéticos, fatores alimentares, questões sócio-ambientais e comportamentais como contribuintes para a gênese da doença, devendo ser considerados em sua amplitude para a prevenção de neoplasias mamárias (Inumaru et al., 2011; Neuber, 2010).

Quando bem estruturada, a mídia pode ter um papel de grande relevância na ampliação do conteúdo e sentido da informação veiculada, permitindo desconstruir alguns mitos sobre a doença que circula no imaginário social. Entre os principais mitos relacionados ao assunto que merecem ser desconstruídos, Garcia et al. (2010) elegem os seguintes: (i) não há nada que se possa fazer para prevenir a doença, o câncer é o destino, não havendo assim a possibilidade de prevenção para a doença; (ii) o câncer é uma sentença de morte, que se refere à crença de não haver tratamento efetivo para a doença e que, independente da fase em que for diagnosticada, não há cura.

Os mitos suprarrelacionados permeiam as representações sociais acerca da doença e tendem a não favorecer a adoção de práticas preventivas e de autocuidado, podendo se tornar um obstáculo na adoção de comportamentos voltados à saúde, dificultando a adesão de práticas e ações voltadas para a prevenção do câncer de mama, apesar das inúmeras publicações relacionadas ao incentivo e importância das práticas de prevenção (GiraldoMora \& Rojas, 2009).

$\mathrm{Na}$ comunicação midiática acerca do câncer de mama, pôde-se perceber que os conteúdos veiculados indicavam mais do que a mera transmissão de informações, mas o compartilhamento de representações sobre a doença, sendo a comunicação uma forma de promover a ressignificação da mensagem que vai sendo alterada em função de normas e valores coletivos (Moscovici, 2012; Vala, 2013). 


\section{Conclusões}

Considerando que a mídia possui um papel de destaque na construção do conhecimento do senso comum, o presente estudo objetivou apreender as representações sociais do câncer de mama que são veiculadas pela mídia impressa, especificamente, pelas mídias, nacional (Folha de São Paulo) e local (Correio da Paraíba).

Conforme a literatura especializada preconiza, a noção de prevenção do câncer de mama transpõe a prática de cuidado estritamente ligada à realização de exames periódicos, contemplando hábitos saudáveis como estratégias preventivas igualmente importantes. Entretanto, de acordo com a análise das matérias veiculadas pelos jornais aqui considerados, foi possível identificar uma valorização hegemônica do enfoque médico, em detrimento de outros aspectos envolvidos na gênese da doença.

Embora a comunicação midiática possa, ao veicular informações acerca do câncer de mama, assumir uma importante função na prevenção desta doença, os resultados da pesquisa indicaram a necessidade de se atentar à natureza do conteúdo que é veiculado. Isto é, os achados destacaram que, ao contrário do que se pretende, a ênfase em notícias com dados sobre índice de mortalidade e outras considerações científicas pode ter um efeito repulsivo, uma vez que a doença fica associada à morte como realidade próxima, contribuindo para cristalizar o estigma associado ao câncer de mama, deixando de ter um efeito convidativo para práticas de autocuidado e detecção precoce da doença.

Nesta perspectiva, sugere-se uma reflexão em torno do processo de comunicação que envolve a construção e transformação das representações sociais sobre o tema de interesse. Por exemplo, apesar de a mídia funcionar como exímia difusora de conteúdos do saber científico para o senso comum, este veículo de comunicação, a fim de abordar o tema do câncer de mama em suas múltiplas facetas, pode se abrir a pautas que potencialmente surjam das conversações do cotidiano, dando visibilidade ao assunto a partir de relatos de atores sociais que vivenciaram e trataram a doença, contribuindo para desconstruir representações cristalizadas sobre o caráter fatal do câncer.

Assim, os conteúdos veiculados pela mídia de massa, considerada de grande valia na construção das representações sociais acerca do câncer de mama e na luta contra a doença, podem ser apontados como um fator transformador ao facilitar a comunicação entre os grupos e orientar práticas e comportamentos de autocuidado. Ao partilhar a informação e contribuir com a formação do conhecimento, a mídia assume um lugar de destaque na elaboração e reelaborações das representações acerca de um dado objeto social. Neste estudo sobre as representações sociais midiáticas, ficaram evidentes as possibilidades de ações terapêuticas e preventivas em relação à doença que podem tomar os espaços jornalísticos.

Ademais, ainda no domínio das representações sociais, recomendam-se pesquisas sobre as representações sociais do câncer de mama na perspectiva de atores sociais com e sem histórico de neoplasia mamária, a fim de conhecer o caráter prático que a doença adquire em suas vidas, determinando ações e condutas no cotidiano e nas práticas de cuidado em saúde.

Apesar das limitações do estudo, considerando o objetivo inicialmente proposto nesta pesquisa - conhecer o conhecimento difundido pela mídia impressa sobre o câncer de mama em mulheres -, acredita-se que este foi alcançado. Salienta-se, no entanto, que os resultados da pesquisa não são conclusivos e não devem ser generalizados, pois se fundamentam em 
documentos de apenas dois jornais. Por outro lado, sugerem-se estudos futuros com o objetivo de ampliar as fontes de busca, ou seja, outras mídias impressas e, quiçá, televisivas.

Finalmente, confia-se que os achados serviram para explicar a difusão do câncer de mama pela mídia impressa, tornando notória a necessidade de inclusão de pautas de comunicação positivas sobre o tema e suas possíveis implicações nas práticas de prevenção e cuidado no contexto oncológico.

\section{Referências}

Allain, J. M., \& Camargo, B. V. (2007). O papel da mídia brasileira na construção das representações sociais de segurança alimentar. Psicologia: teoria e prática, 9(2), 92-108.

Bauer, M. (1994). A popularização da ciência como imunização cultural: A função das representações sociais. In S. Jovtchelovitch \& P. Guareschi. (Orgs.). Textos em representações sociais (pp. 229-257). Petrópolis: Vozes.

Castro, R. (2009). Câncer na mídia. Uma questão de saúde pública. Revista Brasileira de Cancerologia, 55(1), 4148.

Coutinho, M. P. L., \& Saraiva, E. R. A. (2013). Teoria das Representações Sociais. In N. T. Alves, J. M. Andrade, I. F. Rodrigues \& J. B. Costa (Eds.). Teoria das Representações Sociais (pp. 73-114). João Pessoa: Editora da UFPB.

Feldman, A. F. (2008). Análise das campanhas de comunicação sobre câncer de mama: um estudo comparativo entre as iniciativas do INCA e do IBCC. Dissertação de Mestrado, Escola de Comunicações e Artes/USP, São Paulo, Brasil. Recuperado em 10 fevereiro, 2015, de http://www.teses.usp.br/teses/disponiveis/27/27154/tde-11052009-151132/pt-br.php.

Garcia, C. L., Pereira, H. C., \& Marinho, M. N. A. S. B. (2010). Percepções das mulheres acerca do exame de prevenção do câncer cérvico-uterino. Revista brasileira em promoção da saúde, 23(3), 118-125.

Giraldo-Mora, C. V. (2009). Persistencia de las representaciones sociales del câncer de mama. Revista de Salud Pública, 11(4), 514-525.

Giraldo-Mora, C. V., \& Rojas, M. E. A. (2009). Social representations towards self- care prevention. Investigación y Educación en Enfermeria, 27(2), 191- 200.

INCA-Instituto Nacional do Câncer (2014). Estimativa 2014: incidência do câncer no Brasil. Rio de Janeiro. Recuperado em 05 janeiro, 2014, de http://www.inca.gov.br/estimativa/2014/.

Inumaru, L. E., Silveira, E. A., \& Naves, M. M. V. (2011). Fatores de risco e proteção para câncer de mama: uma revisão sistemática. Caderno de Saúde Pública, 27(7), 1259-1270.

Jodelet, D. (2001). As representações sociais. Rio de Janeiro: EDUERJ.

Jurberg, C, Gouveia, M. E., \& Belisário, C. (2006). Na mira do câncer: o papel da mídia brasileira. Revista Brasileira de Cancerologia, 52(2), 139-146.

Kluthcovsky, A. C. G. C., Faria, T. N. P., Carneiro, F. H., \& Strona, R. (2014). Female breast cancer mortality in Brazil and its regions. Revista da Associação Médica Brasileira, 20(4), 387-393.

Lara, M. A. (2008). A construção da imagem do câncer na mídia. Apresentação de ppt: 2008. Recuperado em 10 fevereiro, 2015, de http://www4.ensp.fiocruz.br/biblioteca/dados/txt_579302582.ppt.

Moscovici, S. (2011). Representações sociais: investigações em psicologia social. Petrópolis: Vozes.

Moscovici, S. (2012). A Psicanálise, sua Imagem e seu Público. Petrópolis: Vozes.

Neuber, L. M. B. (2010). Sociodrama e prevenção do câncer de mama em mulheres com conflitos conjugais e familiares. Tese de doutorado, Faculdade de Medicina de Botucatu, Botucatu, Brasil. Recuperado em 08 janeiro, 2015, de 
http://base.repositorio.unesp.br/bitstream/handle/11449/106363/neuber_Imb_dr_botfm.pdf?sequenc $\mathrm{e}=1$ \&isAllowed=y.

Oliveira, D. C. (2011). A teoria das representações sociais como grade de leitura da saúde e da doença. In A. M. O. Almeida, M. F. S., \& Santos e Z. A. Trindade (Eds.). Teoria das representações sociais: 50 anos (pp. 585-624). Brasília:Technopolitik.

Quintana, A. M., Borges, Z. N., Tonetto, A. M., Oliveira, D. S., Weber, B. T., \& Russowsky, I. L. T. (2004). Prevenção do câncer de mama: a contribuição das representações sociais. Ciência, Cuidado e Saúde Maringá, 3(3), 295-302.

Rodríguez-Sales, V., Ortiz-Barreda, G., \& Sanjosé, S. (2014). Revisión bibliográfica sobre la prevención del cáncer en personas inmigrantes residentes en España. Revista Española de Salud Pública, 88(6), 735-743.

Saraiva, E. R. A. (2010). Violência contra idosos: aproximações e distanciamentos entre a fala do idoso e o discurso da mídia impressa. Tese de doutorado, Universidade Federal da Paraíba, João Pessoa, Brasil. Recuperado em 18 junho, 2011, de http://bdtd.biblioteca.ufpb.br/tde_busca/arquivo.php?codArquivo=936.

Saraiva, E. R. A., \& Coutinho, M. P. L. (2012a). A difusão da violência contra idosos: um olhar psicossocial. Psicologia \& Sociedade, 24(1), 112-121.

Saraiva, E. R. A., \& Coutinho, M. P. L. (2012b). Meios de comunicação impressos, representações sociais e violência contra idosos. Psicologia em Estudo, 17(2), 205-214.

Saraiva, E. R. A, Coutinho, M. P. L., \& Miranda, R. S. (2011). O emprego do software Alceste e o desvendar do mundo lexical em pesquisa documental. In M. P. L. Coutinho \& E. R. A. Saraiva (Org.).Métodos de pesquisa em psicologia social: perspectivas qualitativas e quantitativas (pp. 67-94). João Pessoa: Ed. Universitária.

Silva, J. P., Bousfield, A. B. S., \& Cardoso, L. H. (2014). A hipertensão arterial na mídia impressa: análise da revista Veja. Psicologia \& Saber Social, 2(2), 191-203.

Soares, P. B. M., Carneiro, J. A., Rocha, L. A., Gonçalves, R. C. R., Martelli, D. R. B., Silveira, M. F. et al. (2013). The quality of life of disease-free Brazilian breast cancer survivors. Revista da Escola de Enfermagem da USP, 47(1), 69-75.

Tavares, J. S. C., \& Trad, L. A. B. (2005). Metáforas e significados do câncer de mama na perspectiva de cinco famílias afetadas. Cadernos de saúde pública, 21(2), 426-435.

Vala, J. (2013). Pensamento social e representações sociais. In J. Vala \& M. B. Monteiro (Eds.). Psicologia social (pp. 353-384). Lisboa: Calouste Gulbenkian. 